\title{
Capital relacional y crecimiento empresarial: efecto indirecto de la capacidad de asimilación en las empresas textiles y de confecciones
}

Relational capital and business growth: indirect effect of the assimilation capacity in textile and clothing companies a characterization in a multiple case study

\author{
Bernardo De la Gala-Velásquez \\ Universidad Nacional San Agustín de Arequipa \\ bdelagala@unsa.edu.pe \\ Américo Hurtado-Palomino \\ Universidad Nacional San Agustín de Arequipa \\ ahurtado@unsa.edu.pe \\ Angela Arredondo-Salas \\ Universidad Nacional San Agustín de Arequipa \\ aarredondos@unsa.edu.pe
}


La competitividad y el dinamismo de la industria textil exigen a las organizaciones desarrollar diversas estrategias para mejorar su crecimiento en el mercado. En este contexto, el capital relacional y la capacidad de asimilación del conocimiento juegan un rol importante para el crecimiento. El objetivo del estudio es analizar el efecto indirecto de la capacidad de asimilación en la relación entre capital relacional y crecimiento empresarial. La investigación se realizó sobre una muestra de 131 empresas textiles y de confecciones en Arequipa, mientras que para el análisis estadístico se aplicaron ecuaciones estructurales mediante mínimos cuadrados parciales (PLS). Del análisis, se concluye que el capital relacional tiene un efecto positivo en el crecimiento empresarial y que, además, la capacidad de asimilación del conocimiento tiene un rol mediador en la relación. Estos resultados contribuyen al desarrollo teórico y práctico de las empresas textiles.

Palabras clave: capacidad de asimilación, capital social relacional, crecimiento empresarial, empresa textil

The competitiveness and dynamism of the textile industry requires organisations to develop various strategies to improve their growth in the market. In this context, relational capital and the ability to assimilate knowledge play an important role for growth. The objective of this study is to analyse the indirect effect of assimilation capacity on the relationship between relational capital and business growth. The research was carried out on a sample of 131 textile and garment companies from Arequipa, using partial least squares (PLS) structural equations for the statistical analysis. From the analysis, it is concluded that relational capital has a positive effect on business growth and that, in addition, the capacity to assimilate knowledge has a mediating role in the relationship. These results contribute to the theoretical and practical development of textile companies. 


\section{Introducción}

El incremento de la competitividad y los desafíos por lograr la sostenibilidad de las micro y pequeñas empresas de la industria textil exigen desarrollar diversas estrategias empresariales operativas y de gestión, aprovechando sus recursos y capacidades (Israel \& Hitzeroth, 2018; Bunclark \& Barcellos-Paula, 2021). En este contexto, el capital relacional y la capacidad de asimilación del conocimiento se convierten en factores clave del éxito para el crecimiento de las organizaciones.

En el Perú, el sector textil es una de las principales industrias no extractivas, pues contribuye con el 1,3\% del producto bruto interno (PBI) (Cortez et al., 2020) y representa el $13,30 \%$ de la producción manufacturera (Andrade et al., 2019). Además, crea un aproximado de 412000 empleos directos e indirectos en toda la población económicamente activa, según el Ministerio de la Producción del Perú. La actividad manufacturera de la industria textil y dedicada a las confecciones tradicionalmente se ha concentrado en Lima (Produce, 2015). Mientras tanto, Arequipa es considerada como la segunda ciudad con mayor número de micro y pequeñas empresas textiles del país, resaltando su importancia en la generación de empleo y para el dinamismo económico en la zona sur del Perú (Ødegaard, 2017). La cantidad de empresas activas que conforman el tejido empresarial permite inferir la potencialidad para desarrollar un clúster y generar competitividad regional a través de este sector económico.

Al revisar la literatura, se han identificado pocos estudios sobre la gestión empresarial en la industria textil del Perú (Ødegaard, 2017; Andrade et al., 2019; Bunclark \& Barcellos-Paula, 2021). Además, la industria textil ha experimentado los cambios de la competencia global, así como el dinamismo tecnológico y del mercado, lo que exige desarrollar diversas estrategias empresariales para mantenerse y alcanzar un mayor crecimiento en el mercado, especialmente a las empresas localizadas en zonas estratégicas de desarrollo económico regional (Ødegaard, 2017; Israel \& Hitzeroth, 2018). Este escenario ha motivado a explorar las capacidades relacionales de las empresas vinculadas a las redes de contacto con clientes, proveedores y competidores que favorezcan el crecimiento empresarial (Partanen et al., 2008); y, por otro lado, a conocer el efecto directo e indirecto de la capacidad de asimilación del conocimiento en el desarrollo de nuevos productos y servicios (Jeong et al., 2019). Por tanto, el capital relacional y la capacidad de asimilación se convierten en un antecedente para el crecimiento empresarial.

Los estudios previos sostienen los vínculos teóricos y prácticos entre el capital relacional y el crecimiento empresarial en diferentes sectores económicos (Arshad \& Arshad, 2019; Expósito-Langa et al., 2015; Martins, 2016). En la industria manufacturera existen estudios cercanos que analizan el capital relacional con el resultado empresarial (Partanen et al., 2008; Yu \& Huo, 2019; Yu et al., 2021). Asimismo, en las empresas textiles se han identificado las investigaciones de Murale et al. (2018) y Mubarik et al. (2019); no obstante, quedan vacíos en la literatura sobre los efectos indirectos de los factores internos y externos (Safrida et al., 2020). En esta línea, la capacidad de asimilación puede ser el camino que mejorará el crecimiento empresarial por su alto potencial de análisis, procesamiento, interpretación y comprensión del conocimiento adquirido en un entorno dinámico. Esta disyuntiva nos lleva a formular la siguiente pregunta de investigación: 
¿cuál es el efecto mediador de la capacidad de asimilación en la relación entre el capital social relacional y el crecimiento empresarial de las empresas textiles?

En resumen, el objetivo de la investigación es analizar el efecto mediador de la capacidad de asimilación en la relación entre el capital relacional y el crecimiento empresarial de las empresas textiles. Con los resultados de la investigación se pretende contribuir a profundizar y ampliar la base teórica de la conexión entre el capital relacional y el rendimiento empresarial. Además, el trabajo busca aportar una mejor comprensión del efecto indirecto de la capacidad de asimilación de las empresas textiles. En cuanto a la contribución práctica, busca comprender la importancia del capital relacional y la capacidad de asimilación como determinantes directos e indirectos del crecimiento empresarial para utilizar óptimamente los recursos y capacidades organizacionales.

Este trabajo de investigación inicia con esta introducción y prosigue con el desarrollo del marco teórico y las hipótesis, donde se explican las bases teóricas y relaciones entre las variables de estudio. A continuación, se describe la metodología utilizada. Finalmente, se presentan los resultados, la discusión y las conclusiones.

\section{Revisión de teoría e hipótesis}

\subsection{Crecimiento empresarial}

A lo largo del desarrollo de la literatura, el crecimiento empresarial fue estudiado desde diferentes enfoques: estocástico, descriptivo, evolutivo, de recursos y capacidades, de aprendizaje y determinista (Dobbs \& Hamilton, 2007). En este trabajo se analiza el crecimiento empresarial bajo el enfoque de la teoría de los recursos y las capacidades (Penrose, 1959), bastante estudiado en el ámbito de la gestión empresarial por su contribución a la ventaja competitiva y sostenibilidad de los negocios en entornos dinámicos (Kor \& Mahoney, 2004).

Posteriormente, el crecimiento empresarial ha sido estudiado en diferentes ámbitos organizacionales, generándose contribuciones teóricas y empíricas (Miroshnychenko et al., 2021). Gielnik et al. (2017) consideran que el crecimiento de las empresas está caracterizado por la diversidad conceptual, complejidad y multidimensionalidad. Esta diversidad conceptual se produce por los diferentes enfoques e indicadores utilizados para su medición (Dobbs \& Hamilton, 2007). En esta línea, García-Villaverde et al. (2014) sostienen que el crecimiento empresarial se entiende como el incremento de la cuota de mercado y el crecimiento de las ventas. Por otro lado, en la práctica empresarial, el crecimiento empresarial es el reflejo o resultado de las acciones emprendedoras.

\subsection{Capital relacional}

El capital relacional nace como una dimensión de la teoría del capital social y (Nahapiet \& Ghoshal, 1998), desde entonces, ha tenido una amplia aceptación en el campo empresarial. Rodrigo-Alarcón et al. (2014) sostiene que el capital social es el «conjunto de relaciones entre individuos y organizaciones que facilitan la acción y ofrecen oportunidades a los 
miembros de una red social» (p. 133). Asimismo, Nahapiet y Ghoshal (1998) plantean tres dimensiones: estructural, relacional y cognitiva, que miden el capital social. Para este estudio se ha tomado la dimensión del capital relacional, que se caracteriza por el respeto, confianza, amistad y confiabilidad con la red de relaciones personales u organizacionales (García-Villaverde et al., 2010; Rodrigo-Alarcón et al., 2014). De igual manera, GarcíaVillaverde et al. (2018) considera que el capital relacional se centra en la motivación existente para interactuar e intercambiar información entre las empresas.

\subsection{Capacidad de asimilación}

Los antecedentes de la literatura sobre la capacidad de absorción han tomado relevancia en el ámbito académico y empresarial. Esta teoría fue estudiada con diferentes dimensiones, como las de capacidad de adquisición, asimilación del conocimiento, transformación y explotación (Cohen \& Levinthal, 1990). En relación a la capacidad de asimilación del conocimiento, Rodrigo-Alarcón (2013) sostiene que esta es entendida como «las rutinas y procesos de la organización que le permiten analizar, procesar, interpretar y comprender la información obtenida del exterior» (p. 157). Es decir, las organizaciones pueden adquirir conocimientos externos que luego deben ser asimilados o interiorizados porque, de lo contrario, no representarían ningún beneficio para la misma (García-Villaverde et al., 2014).

\subsection{Capital relacional y crecimiento empresarial}

La interacción constante de información entre las empresas y su entorno exige establecer relaciones para adquirir, asimilar, transformar y explotar los conocimientos esenciales en beneficio de las organizaciones (Murale et al., 2018). En la industria manufacturera, Yu y Huo (2019) examinan el capital relacional, la integración de la calidad y el desempeño operativo, concluyendo que el capital relacional tiene un efecto positivo sobre el desempeño operativo, mientras que la integración de la calidad tiene un rol mediador total. Posteriormente, Yu et al. (2021) han concluido que el capital relacional de proveedores y clientes mejora el desempeño financiero a través de la gestión logística verde. Asimismo, Prajogo et al. (2021) sostienen que las organizaciones pueden tener beneficios significativos si las empresas construyen colaboraciones estratégicas. Además, se infiere que el capital relacional, a través del compromiso y la confianza, contribuye en las colaboraciones estratégicas y el rendimiento de las empresas manufactureras.

Sin embargo, existe escasa literatura que vincule el capital relacional con el crecimiento empresarial en empresas textiles y dedicadas a las confecciones, aunque sí hay estudios muy cercanos. Por ejemplo, Mubarik et al. (2019) analizan el capital relacional en una cadena de suministro basándose en los proveedores para mejorar el desempeño logístico de una empresa del sector textil, donde los resultados alcanzados sostienen una relación positiva y significativa. Asimismo, Murale et al. (2018) estudian el capital relacional y el desempeño empresarial en la industria textil de la India, donde se concluye la relación positiva entre las variables. 
Concretamente, es natural que las empresas establecidas en un sector económico maduro, como el textil, establezcan una red de relaciones personales e interorganizacionales con el resto de los agentes, generando respeto, confianza, amistad y confiabilidad (Murale et al., 2018), lo que permite alcanzar mejores resultados de crecimiento empresarial a través del incremento de la cuota de mercado y el crecimiento de las ventas. Después de revisar los diferentes argumentos, proponemos la siguiente hipótesis de investigación:

H1: el capital relacional tiene una influencia positiva en el crecimiento empresarial.

\subsection{Efecto indirecto de la capacidad de asimilación}

El dinamismo del mercado ha introducido el término "coopetencia» en el ámbito de la gestión empresarial, lo que lleva a pensar que las empresas por sí solas no pueden crear todos los recursos y capacidades para prosperar y crecer (Yoo et al., 2016). En ese contexto, es necesario analizar la interacción del capital relacional y la capacidad de asimilación. Asimismo, el desglose de la capacidad de absorción en dimensiones presenta una buena oportunidad para analizar con mayor detalle la contribución de cada dimensión al crecimiento empresarial.

Los estudios previos consideran que la capacidad de absorción puede permitir un mejor resultado comercial a partir de la red de relaciones de una empresa (Hughes et al., 2014). Por su parte, Debrulle et al. (2013) estudian la conexión entre el capital social y la capacidad de absorción a través de sus dimensiones, concluyendo que el capital social está relacionado positivamente con la capacidad de las empresas para adquirir, asimilar y explotar información externa. De estos resultados se infiere que existe relación entre el capital social relacional y la capacidad de asimilación del conocimiento.

En cuanto a la relación entre la capacidad de asimilación del conocimiento y el crecimiento empresarial, se han encontrado investigaciones que analizan la capacidad de absorción como una variable de segundo orden, de forma directa o indirecta. En esta línea, Kohtamäki et al. (2019) estudian el efecto moderador de la capacidad de absorción en la relación entre la orientación emprendedora y el crecimiento empresarial. Las conclusiones más relevantes del estudio sostienen que los «procesos y rutinas [...] facilitan la adquisición, asimilación, transformación y explotación» (p. 102), y tienen un efecto indirecto en el crecimiento de las ventas. Por otro lado, Liu et al. (2018) examinan la relación entre la capacidad de absorción y el desempeño empresarial, con efectos indirectos sobre la innovación y la personalización en masa, de cuyos resultados se puede inferir que la capacidad de absorción mejora directamente el desempeño empresarial. Además, Camisón y Forés (2010) sostienen que la capacidad de asimilación del conocimiento se convierte en un factor clave para el desarrollo de nuevos productos, la adaptación al entorno y el crecimiento empresarial. A partir de estos argumentos, se ha planteado la siguiente hipótesis de investigación:

H2: el capital relacional tiene una influencia indirecta y positiva a través de la capacidad de asimilación en el crecimiento empresarial. 


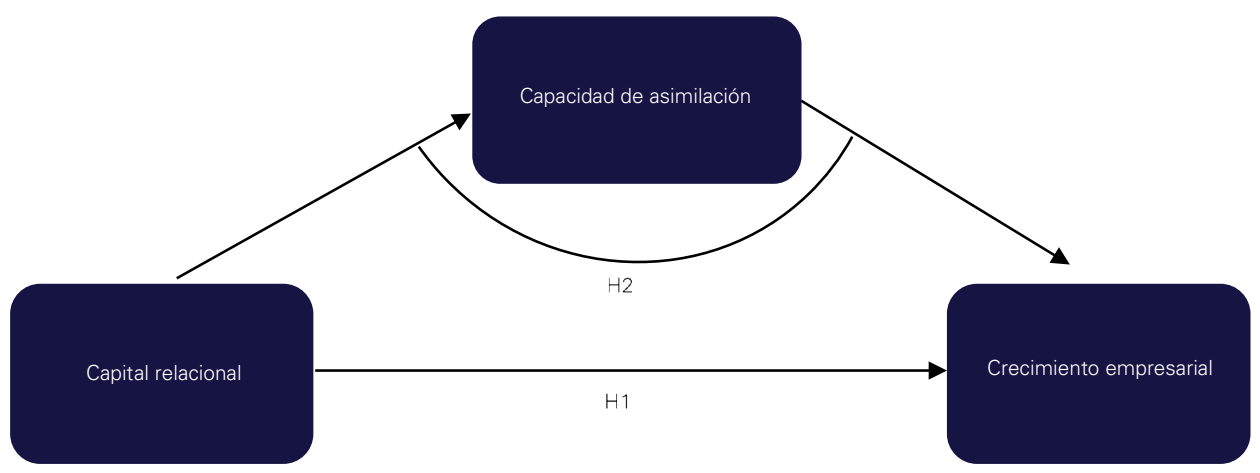

Fuente: elaboración propia.

La figura 1 explica el modelo teórico de investigación, donde H1 (+) representa la influencia directa del capital social relacional y el crecimiento empresarial. La hipótesis H2 $(+)$, por su parte, representa el efecto indirecto del capital social relacional en el crecimiento empresarial a través de la capacidad de asimilación.

\section{Metodología}

\subsection{Población y muestra}

Según la Superintendencia de Administración Tributaria del Perú (Sunat) (2020), existen 10633 empresas activas del sector textil y confecciones, de las cuales aproximadamente 508 corresponden a dicho sector en la región Arequipa (ver figura 2).

Figura 2. Empresas activas del sector textil y confecciones

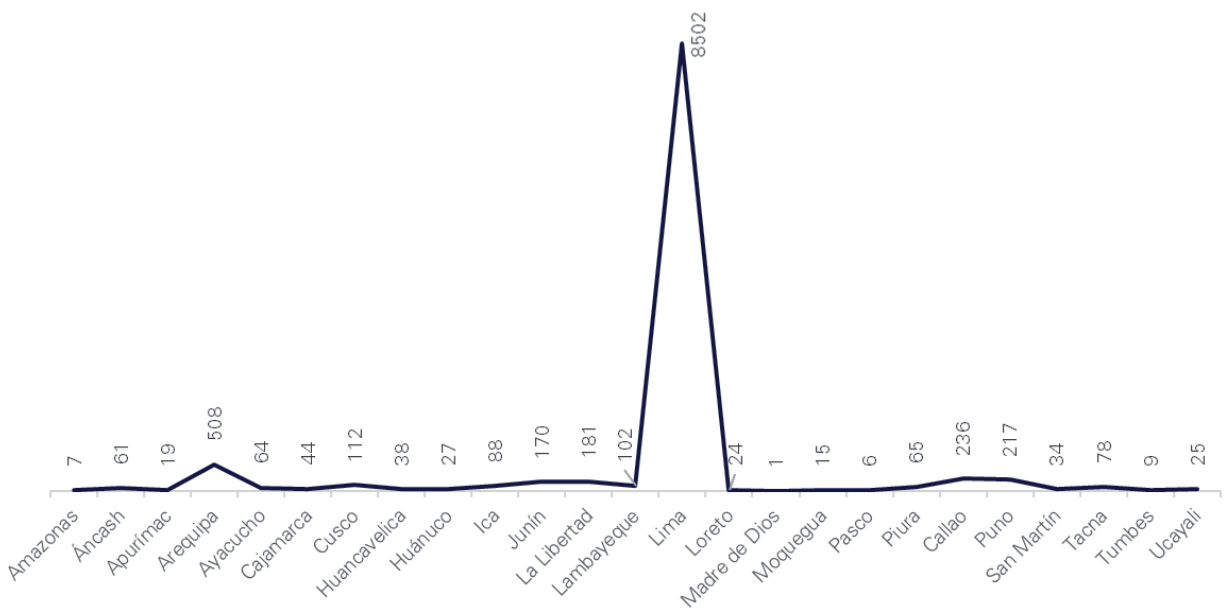

Fuente: elaborado a partir de información de la Sunat (2020) obtenida mediante una solicitud de acceso a la información pública. 
La figura 2 muestra la cantidad de empresas del sector textil y confecciones activas en el Perú. Asimismo, destaca Lima, que concentra el 79,96 \% de las empresas; seguida por la región Arequipa, que representa el 4,78 \%. Luego, destaca la contribución de la región Puno $(2,04 \%)$ y de la provincia constitucional del Callao $(2,22 \%)$.

Tabla 1. Empresas registradas en la Sunat - región Arequipa

\begin{tabular}{l|c|c} 
Descripción & Número de empresas & Porcentaje \\
\hline Activo & 508 & $37,8 \%$ \\
\hline Baja de oficio & 720 & $1,5 \%$ \\
\hline Baja definitiva & 20 & $0,2 \%$ \\
\hline Baja múltiple de inscripción & 3 & $0,7 \%$ \\
\hline Baja provisional por oficio & 9 & $6,2 \%$ \\
\hline Suspensión temporal & 83 & $\mathbf{1 0 0} \%$ \\
\hline Total & $\mathbf{1 3 4 3}$ &
\end{tabular}

Fuente: elaborado a partir de información de la Sunat (2020) obtenida mediante una solicitud de acceso a la información pública.

La tabla 1 muestra 1343 empresas registradas en la Sunat para la región Arequipa; sin embargo, solo el 37,8 \% representa a las empresas activas a enero del 2020. Asimismo, el 53,6 \% de las empresas tiene baja de oficio, mientras que un 6,2 \% tiene suspensión temporal. En general, se infiere que el 62,2 \% de las empresas constituidas y registradas han fracasado por diversas razones.

Para los propósitos de este estudio, excluimos a las empresas con menos de un año, dado que necesitan ese periodo de tiempo para desarrollar relaciones interorganizacionales y otras capacidades. Después de aplicar los filtros correspondientes, se cuenta con una población de 418 empresas. Luego, se utilizó un muestreo por conveniencia debido a que no todas las empresas estaban dispuestas a participar en el estudio, obteniendo una muestra de 131 empresas, que representan el 31,34 \% de la población total. Se administró una encuesta estructurada en cuatro secciones: la primera sección recopilaba datos sobre las variables de control; la segunda sección, sobre capital relacional; la tercera, sobre capacidad de asimilación; y la última, sobre el crecimiento empresarial.

Para valorar el tamaño de la muestra y la potencia estadística, se realizó un análisis de tamaño muestral con el software Gpower 3.1 en el que se utilizó el test estadístico de regresión lineal múltiple con un tamaño de efecto medio de 0,15, un $\alpha$ de 0,05 y una potencia estadística de 0,95. Así, se obtuvo un tamaño de muestra de 129 encuestas para cuatro predictores, que son el número máximo dentro del modelo. Sin embargo, con el mismo efecto medio y una potencia estadística de 0,8, que suele ser la más utilizada (Cepeda et al., 2017), se obtuvo un tamaño de muestra de 85 encuestas, cantidad mucho menor a la trabajada en el análisis del estudio. 


\subsection{Medidas}

Las escalas utilizadas para medir las variables se adaptaron de estudios previos, como producto de la revisión de la literatura. Cada uno de los ítems se midió en una escala tipo Likert de 7 puntos, donde 1 representa "muy en desacuerdo" y 7 equivale a «muy de acuerdo». Todos los ítems utilizados en el cuestionario se incluyen en el apéndice.

- Capital social relacional: se caracteriza por el respeto, confianza, amistad y confiabilidad con la red de relaciones personales u organizacionales (GarcíaVillaverde et al., 2010; Rodrigo-Alarcón et al., 2014; Rodrigo-Alarcón, 2013). Para medir esta variable fueron utilizados cuatro ítems, ampliamente aplicados en trabajos previos (García-Villaverde et al., 2010; Rodrigo-Alarcón et al., 2018). Cabe indicar que en el apéndice se encuentran adjuntos los ítems de la investigación.

- Capacidad de asimilación del conocimiento: en la literatura se han identificado diversos instrumentos que analizan esta dimensión. Para el presente estudio se adaptó el instrumento validado por Flatten et al. (2011), desarrollado a partir del análisis de varios artículos publicados en revistas relevantes y ampliamente aceptado en la literatura (García-Villaverde et al., 2018; Parra-Requena et al., 2015).

- Crecimiento empresarial: el crecimiento es el resultado de las diversas estrategias desarrolladas por la organización para el incremento de la cuota de mercado y crecimiento de las ventas. El instrumento recoge información bajo una escala subjetiva ampliamente utilizada en estudios previos (García-Villaverde et al., 2014). Además, se ha recogido la valoración dada por los gerentes y/o administradores sobre la importancia y la satisfacción de los resultados alcanzados. Para esta variable se ha optado por las medidas propuestas por Rodrigo-Alarcón (2013). Los ítems se pueden observar en el apéndice.

\subsection{Variables de control}

En el estudio se ha considerado como variables de control el tamaño de la empresa y la municipalidad en la que están ubicadas las empresas textiles. Ambas variables tienen una relación significativa con el crecimiento empresarial con coeficientes path de $0,104^{* *}$ y $-0,146^{* *}$, respectivamente. De acuerdo con Hjalager (2010), las municipalidades son espacios donde se acumulan competencias y conocimientos únicos e inimitables para el desarrollo de productos y servicios, generando un mayor crecimiento empresarial por las características del mercado y la cercanía a los recursos. Por otra parte, el tamaño de las empresas caracterizado por el número de trabajadores (Rodrigo-Alarcón et al., 2020) sugiere, además, que a mayor tamaño existe un mayor crecimiento empresarial.

\subsection{Procedimiento y técnicas de análisis}

El recojo de la información se realizó de manera personal con los directivos de las empresas del sector textil en la región Arequipa mediante un cuestionario de once ítems, cinco de 
ellos correspondientes al capital social relacional, cuatro a la capacidad de asimilación del conocimiento y dos a la variable crecimiento empresarial. Esta información fue analizada con el modelamiento de ecuaciones estructurales (SEM) basadas en la varianza y aplicando la técnica de mínimos cuadrados parciales (PLS), muy utilizada en investigaciones de ciencias sociales (Chin, 1998).

De acuerdo a Hair et al. (2019) los investigadores deben utilizar PLS-SEM cuando el modelo estructural es complejo y con muchas construcciones, y además sirven para explorar extensiones teóricas de las variables ya establecidas. El presente estudio tiene tres variables donde se muestran relaciones directas e indirectas que tratan de explorar extensiones teóricas del capital relacional y la capacidad de asimilación, y cómo se relacionan con el crecimiento empresarial.

Igualmente, existen dos enfoques clásicos para evaluar las relaciones que aparecen en un modelo SEM (Hair et al., 2019). Por un lado, el CB-SEM analiza las variables latentes como modelos de factor común, y es un enfoque muy difundido y utilizado para confirmar teorías. Por otro, el PLS-SEM utiliza compuestos ponderados de los indicadores relacionados con cada constructo (Hair et al., 2017), motivo por el que constituye un enfoque basado en compuestos. Además, es adecuado cuando la finalidad del estudio es la explicación de los constructos (Rigdon, 2012).

El análisis se realizó utilizando el software de ecuaciones estructurales PLS Smart, versión 3.3.3, que ha demostrado eficacia cuando las investigaciones en ciencias sociales contrastan modelos complejos (Avkiram, 2018). Además, se ha constatado que este programa tiene altos niveles de potencia estadística con marcos muestrales pequeños, siendo muy bondadoso en sus resultados con muestras grandes. Por otro parte, en el estudio se utiliza el modelo de compuestos, que son calculados mediante un algoritmo que se obtiene de una combinación lineal de diversos indicadores, los cuales son valorados mediante una serie de pesos, multiplicados por sus observaciones asociadas a las variables y, posteriormente, sumados. Además, el valor compuesto es calculado para cada uno de los encuestados de la muestra (Hair et al., 2017).

La modelización de ecuaciones estructurales con PLS-SEM tiene dos elementos. El primero es conocido como el modelo de medida, que refleja las relaciones de los constructos con sus indicadores. Este modelo se analiza mediante el de medida de variables latentes exógenas, que valora las relaciones de las variables exógenas con sus indicadores. El otro modelo de medida es el de variables latentes endógenas, que valora las relaciones de las variables endógenas con sus indicadores. El segundo elemento es el modelo estructural, donde se muestran las relaciones entre los constructos (Hair et al., 2019).

En este sentido, la presente investigación tiene compuestos reflectivos, donde las medidas representan los efectos o manifestaciones de un constructo y la causalidad va desde el constructo hacia a sus medidas; y los indicadores deberán de ser altamente correlacionados y pueden ser intercambiables (Hair et al., 2017).

El estudio analizó la información en tres etapas: la primera correspondió a una evaluación descriptiva de las variables de estudio y control; a continuación, se realizó una valoración del modelo de medida con que se evalúa la fiabilidad y validez de las variables, así como la colinealidad en sus indicadores; y, posteriormente, se evaluó el 
modelo estructural, con el que se estiman las relaciones directas e indirectas mediante el bootstrapping, en función al modelo teórico propuesto de este sistema de ecuaciones (Roldan \& Sánchez-Franco, 2012).

\section{Resultados}

\subsection{Resultados descriptivos}

Los resultados de la estadística descriptiva describen la media, la desviación estándar, la asimetría, el factor de inflación de la varianza (VIF, por sus siglas en inglés) y las correlaciones de las variables analizadas. En el caso de la asimetría, se observan valores menores a 1 , lo que asegura que no existe una asimetría excesiva que produzca sesgos en el análisis. Además, si bien es cierto que existen valores altos en las correlaciones, estas no muestran colinealidad con valores VIF entre 1 y 3 (Hair et al., 2019).

Tabla 2. Análisis descriptivo de las principales variables de control

\begin{tabular}{l|c|c|c|c|c|c|c|c|c} 
& Media & SD & $\begin{array}{c}\text { Asime- } \\
\text { tría }\end{array}$ & VIF & $\mathbf{1}$ & $\mathbf{2}$ & $\mathbf{3}$ & $\mathbf{4}$ & $\mathbf{5}$ \\
\hline 1. Tamaño & 6,618 & 4,187 & 2,46 & 1,01 & - & & & & \\
\hline 2. Municipalidad & 3,794 & 2,326 & 0,801 & 1,20 & $-0,174^{*}$ & - & & & \\
\hline $\begin{array}{l}\text { 3. Capital } \\
\text { relacional }\end{array}$ & 5,176 & 0,925 & $-0,013$ & 1,36 & $-0,037$ & $-0,172^{*}$ & - & & \\
\hline $\begin{array}{l}\text { 4. Asimilación del } \\
\text { conocimiento }\end{array}$ & 4,96 & 0,924 & 0,001 & 1,50 & 0,165 & $-0,297^{* *}$ & $0,494^{* *}$ & - & \\
\hline $\begin{array}{c}\text { 5. Crecimiento } \\
\text { empresarial }\end{array}$ & 29,13 & 10,09 & 0,201 & & 0,153 & $-0,341^{* *}$ & $0,593^{* *}$ & $0,510^{* *}$ & -
\end{tabular}

Fuente: elaboración propia.

\subsection{Evaluación del modelo de medida}

La fiabilidad y validez de las variables son el principal objetivo que se persigue en un modelo de medida. En esta investigación, la fiabilidad ha sido evaluada usando la fiabilidad compuesta y el alfa de Cronbach, y en ambos casos se observan valores > a 0,7 como nivel de aceptación, comprobándose su consistencia interna (Chin, 1998; Fornell \& Larcker, 1981). Respecto a la validez convergente, encontramos valores $>$ a 0,5 de varianza media extraída, demostrándose su validez convergente (Fornell \& Larcker, 1981). En la validez convergente se utilizaron los criterios de Fornell y Larcker y el hetero-trait mono-trait (HTMT) que se muestran en la tabla 3 , donde se aprecia que la diagonal en negrita tiene valores mayores tanto en las filas superiores como inferiores, aceptándose que las variables tienen una validez discriminante. 


\begin{tabular}{l|c|c|c|c|c|c} 
& \multicolumn{2}{|c|}{ Consistencia interna } & \multicolumn{2}{c}{$\begin{array}{c}\text { Validez } \\
\text { convergente }\end{array}$} & \multicolumn{2}{c}{ Validez discriminante } \\
\hline & $\begin{array}{c}\text { Alpha de } \\
\text { Cronbach } \\
>0,7\end{array}$ & $\begin{array}{c}\text { Fiabilidad } \\
\text { compuesta } \\
>0,7\end{array}$ & $\begin{array}{c}\text { AVE } \\
>0,5\end{array}$ & $\mathbf{1}$ & $\mathbf{2}$ \\
\hline $\begin{array}{l}\text { 1. Capacidad de asimilación del } \\
\text { conocimiento }\end{array}$ & 0,722 & 0,826 & 0,543 & $\mathbf{0 , 7 4}$ & 0,67 & 0,67 \\
\hline 2. Capital social relacional & 0,755 & 0,843 & 0,578 & 0,51 & $\mathbf{0 , 7 6}$ & 0,75 \\
\hline 3. Crecimiento empresarial & 0,826 & 0,92 & 0,852 & 0,54 & 0,62 & $\mathbf{0 , 9 2}$
\end{tabular}

Nota: los valores en cursiva y negrita en diagonal hacia abajo corresponden al análisis de validez convergente de Fornell y Larcker; los valores por encima de esa diagonal corresponden al análisis hetero-trait mono-trait (Henseler et al., 2015).

Fuente: elaboración propia.

En la tabla 4 se muestra el análisis de los indicadores de las variables en estudio, observándose una valoración descriptiva mediante la media y la desviación estándar; además, la validez discriminante a nivel de indicadores muestra valores "t» superiores al nivel requerido y el análisis de cargas cruzadas es $>$ a 0,7 (Carmines, 1979). En cuanto al análisis de colinealidad, se muestran valores mayores a 3,3 (Roberts \& Thatcher, 2009).

Finalmente, respecto a la validez discriminante y de colinealidad a nivel de indicadores, podemos señalar - a manera de resumen- que todos los indicadores muestran valores mayores a los niveles de aceptación requeridos para este tipo de estudio.

Tabla 4. Análisis de fiabilidad de los indicadores, validez discriminante y colinealidad

\begin{tabular}{|c|c|c|c|c|c|}
\hline & \multicolumn{2}{|c|}{ Descriptivos } & \multicolumn{2}{|c|}{ Validez discriminante } & \multirow{2}{*}{$\frac{\text { Colinealidad }}{\text { VIF } \leq \mathbf{3}, \mathbf{3}}$} \\
\hline & Media & $\begin{array}{c}\text { Desviación } \\
\text { estándar }\end{array}$ & ut»" & $\begin{array}{c}\text { Cargas cruza- } \\
\text { das }>0,7\end{array}$ & \\
\hline \multicolumn{6}{|l|}{ Capacidad de asimilación } \\
\hline Ítem 1 & 4,885 & 1,322 & 7,317 & 0,725 & 1,471 \\
\hline Ítem 2 & 4,954 & 1,306 & 8,362 & 0,753 & 1,388 \\
\hline Ítem 3 & 5,137 & 1,195 & 18,635 & 0,797 & 1,408 \\
\hline Ítem 4 & 5,863 & 1,175 & 8,405 & 0,666 & 1,287 \\
\hline \multicolumn{6}{|l|}{ Capital relacional } \\
\hline Ítem 1 & 4,763 & 1,233 & 7,206 & 0,551 & 1,216 \\
\hline Ítem 2 & 5,595 & 1,245 & 22,587 & 0,806 & 1,607 \\
\hline Ítem 3 & 5,244 & 1,342 & 19,868 & 0,849 & 1,778 \\
\hline Ítem 4 & 5,099 & 1,051 & 21,264 & 0,800 & 1,561 \\
\hline \multicolumn{6}{|l|}{ Crecimiento empresarial } \\
\hline Ítem 1 & 27,840 & 10,670 & 76,206 & 0,925 & 1,981 \\
\hline Ítem 2 & 30,427 & 11,195 & 51,606 & 0,921 & 1,981 \\
\hline
\end{tabular}




\subsection{Evaluación del modelo estructural}

Las relaciones sugeridas en el modelo teórico han sido valoradas en esta parte de la investigación según la metodología propuesta en el modelamiento de ecuaciones estructurales. Siguiendo a Hair et al. (2017), los modelos estructurales se enfocan en evaluar las relaciones propuestas en el modelo teórico, sean estas directas o indirectas; sin embargo, para realizar esta evaluación se requiere la aceptación del modelo de medida como paso previo a este análisis.

Si bien es cierto que existe solo un modelo estructural para una mejor comprensión del modelo de relaciones indirectas, en un primer momento se ha analizado la relación directa del capital relacional con el crecimiento empresarial; y, luego, se valoró la relación mediadora de la capacidad de asimilación. Los resultados confirman un incremento en el coeficiente de determinación. A continuación, se explica el modelo estructural.

Los resultados de la tabla 5 expresan la relación directa positiva y significativa del capital relacional en el crecimiento empresarial, mostrándonos un efecto directo de 0,560 con un «t» valor de $10,973^{* * *}$, confirmando la influencia que tiene el capital relacional en el crecimiento empresarial. Además, en la tabla 6 encontramos un coeficiente de determinación $\left(R^{2}=0,430^{* *}\right)$ que es muy significativo, determinándose un alto nivel de influencia del capital relacional en el crecimiento empresarial.

Tabla 5. Análisis del modelo de relación directa

\begin{tabular}{l|c|c|c|c|c} 
Relación & $\begin{array}{c}\text { Efecto } \\
\text { directo }\end{array}$ & $\begin{array}{c}\mathbf{9 5} \% \\
\text { intervalo de } \\
\text { confianza }\end{array}$ & “t” valor & $\begin{array}{c}\text { Significancia } \\
\mathbf{( p < 0 , 0 5 )}\end{array}$ & Condición \\
\hline $\begin{array}{l}\text { Capital relacional } \rightarrow \text { crecimiento } \\
\text { empresarial }\end{array}$ & 0,560 & $\begin{array}{c}(0,485- \\
0,654)\end{array}$ & 10,973 & 0,000 & Significativa \\
& & Fuente: elaboración propia.
\end{tabular}

Los resultados sugieren aceptar la hipótesis $\mathrm{H} 1$, con la que se propuso que el capital relacional tiene una influencia directa y positiva en el crecimiento empresarial.

En un segundo momento, se valoró la relación indirecta de la variable asimilación del conocimiento en la relación entre el capital relacional y el crecimiento empresarial. En este sentido, Nitzl et al. (2016) sustentan que la esencia del análisis mediador es la secuencia de relaciones, donde una variable antecedente afecta a otra variable mediadora que después afecta a la variable dependiente; lo que ayuda a explicar cómo ciertos factores impulsan o suprimen una relación directa entre variables. Además, explica que ocurra una mediación parcial cuando el efecto indirecto de la variable mediadora es significativo, así como el efecto directo del antecedente; que se tenga una mediación parcial complementaria si su coeficiente es positivo; y que se tenga mediación parcial competitiva en el caso de que sea negativo.

Los resultados estadísticos demuestran que esta relación indirecta es positiva y significativa; es decir, el capital relacional, además de influir directamente en el crecimiento empresarial, influye indirectamente a través de la capacidad de asimilación del conocimiento, y esta relación indirecta genera un mayor efecto en el crecimiento empresarial. 


\begin{tabular}{|c|c|c|c|c|c|}
\hline Relaciones & Efectos directos & $\begin{array}{l}95 \% \text { intervalo } \\
\text { de confianza }\end{array}$ & $\begin{array}{l}\text { "t»" } \\
\text { valor }\end{array}$ & $\begin{array}{l}\text { Significancia } \\
(p<0,05)\end{array}$ & Condición \\
\hline $\begin{array}{l}\text { Capital relacional } \rightarrow \text { creci- } \\
\text { miento empresarial }\end{array}$ & 0,508 & $(0,405-0,621)$ & 7,685 & 0,000 & Significativa \\
\hline \multirow{2}{*}{$\begin{array}{l}\text { Capacidad de asimilación } \rightarrow \\
\text { crecimiento empresarial }\end{array}$} & 0,237 & $(0,080-0,376)$ & 2,601 & 0,005 & Significativa \\
\hline & $\begin{array}{l}\text { Efectos indi- } \\
\text { rectos }\end{array}$ & $\begin{array}{l}95 \% \text { intervalo } \\
\text { de confianza }\end{array}$ & $\begin{array}{l}\text { "t" } \\
\text { valor }\end{array}$ & $\begin{array}{l}\text { Significancia } \\
(p<0,05)\end{array}$ & Condición \\
\hline $\begin{array}{l}\text { Capital relacional } \rightarrow \\
\text { capacidad de asimilación } \rightarrow \\
\text { crecimiento empresarial }\end{array}$ & 0,120 & $(0,040-0,199)$ & 2,461 & 0,007 & $\begin{array}{l}\text { Mediación } \\
\text { complemen- } \\
\text { taria }\end{array}$ \\
\hline
\end{tabular}

Fuente: elaboración propia.

La tabla 6 nos muestra que el modelo estimado, donde las relaciones directas para el capital relacional y la capacidad de asimilación tienen relaciones positivas y significativas con coeficientes de regresión de $0,508^{* * *}$ y $0,237^{* *}$, y valores «t» de 7,685*** y 2,601**, respectivamente, demuestra su alto nivel de explicación sobre el crecimiento empresarial. Además, la relación indirecta que tiene el capital relacional a través de la capacidad de asimilación presenta un coeficiente de regresión de $0,120^{* *}$ con un valor «t» de 2,461**, mostrando una influencia indirecta positiva y significativa.

Los datos nos ayudan a identificar una mediación complementaria dado que la capacidad de asimilación del conocimiento influye positivamente en el crecimiento empresarial. Por otro lado, todo el modelo tiene un coeficiente de determinación $\left(\mathrm{R}^{2}=\right.$ $\left.0,463^{* *}\right)$, confirmando un incremento de $6,97 \%$ en la influencia sobre el crecimiento empresarial, en comparación a la valoración anterior (relación directa).

La evaluación del modelo estructural nos permite aceptar la hipótesis H2, la cual formuló que el capital relacional tiene una influencia indirecta y positiva en el crecimiento empresarial a través de la capacidad de asimilación.

Tabla 7. Resumen del modelo de investigación

Variable dependiente: crecimiento empresarial

\begin{tabular}{|c|c|c|}
\hline & Directa & Mediación \\
\hline Capital relacional & $0,560 * * *$ & $0,508 * * *$ \\
\hline Capacidad de asimilación & & $0,237 * *$ \\
\hline $\begin{array}{l}\text { Mediación } \\
\text { Capital relacional } \rightarrow \text { capacidad de asimilación del conoci- } \\
\text { miento } \rightarrow \text { crecimiento empresarial }\end{array}$ & & $0,120 * *$ \\
\hline Tamaño & $0,124 * *$ & $0,104 * *$ \\
\hline Municipalidad & $-0,208 * * *$ & $-0,146^{* *}$ \\
\hline$R^{2}$ ajustada & $0,433^{* * *}$ & $0,463 * * *$ \\
\hline Cambio en $\mathrm{R}^{2}$ ajustada & & $0,030 * * *$ \\
\hline${ }^{*} \operatorname{sig} \leq 0,05 ; \quad * * \operatorname{sig} \leq 0,01 ; \quad * * * \operatorname{sig} \leq 0,001$ & & \\
\hline
\end{tabular}


En la tabla 7 y la figura 3 se observan los resultados globales de los modelos. En estas se puede apreciar y comparar los coeficientes de determinación de ambos modelos con un incremento del 6,97 \% en el grado de influencia de la mediación sobre el modelo de relación directa.

Figura 3. Resultados del modelo

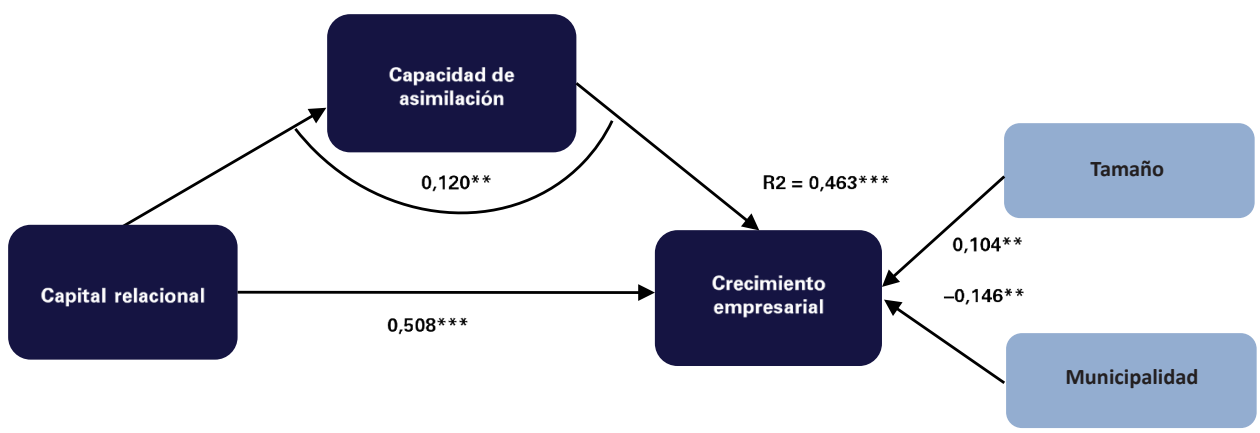

Fuente: elaboración propia.

\section{Discusión de resultados}

La presente investigación tiene como objetivo determinar el efecto indirecto de la capacidad de asimilación en la relación entre el capital relacional y el crecimiento empresarial de las empresas textiles. Después de someter los datos a las diferentes pruebas estadísticas, se ha identificado interesantes aportes teórico y empíricos acerca de estas relaciones, las cuales serán de gran utilidad en el ámbito académico y en la práctica empresarial.

Por un lado, se ha logrado identificar que el capital relacional es un factor determinante para el crecimiento empresarial, caracterizado por el respeto y la confianza, amistad y confiabilidad que se da entre las relaciones de las organizaciones y personas (Murale et al., 2018; Yu \& Huo, 2019; Mubarik et al., 2019; Yu et al., 2021). Además, las relaciones personales se dan con mayor énfasis en pequeños negocios, principal característica de la población en estudio (Ødegaard, 2017), lo que demuestra que dicho rasgo está altamente relacionado con el crecimiento empresarial a través del crecimiento de las ventas y la participación del mercado (Dobbs \& Hamilton, 2007; García-Villaverde et al. 2014); y que las relaciones personales, el respeto y la confianza crean un ambiente adecuado para hacer negocios.

Por otro lado, la capacidad de asimilación del conocimiento, que usualmente ha sido analizada como una dimensión de la capacidad de absorción, está caracterizada por el proceso de análisis, interpretación y comprensión del conocimiento externo a la empresa (Rodrigo-Alarcón, 2013), el cual luego pasa a ser absorbido por la organización en sus rutinas de trabajo. En este sentido, la capacidad de asimilación del conocimiento se convierte en un factor clave al servir de puente entre las relaciones personales e interorganizacionales, permitiendo adquirir el conocimiento externo a la organización; y, si este se asimila de manera adecuada, contribuye al crecimiento empresarial. Cabe añadir que los resultados 
del estudio confirman las investigaciones realizadas anteriormente (Debrulle et al., 2013; Hughes et al., 2014; Kohtamäki et al., 2019).

En concreto, encontramos que el capital relacional influye en el crecimiento empresarial y que este se ve incrementado cuando esta relación se realiza a través de la capacidad de asimilación del conocimiento.

\section{Conclusiones}

La literatura revisada nos dio a conocer que parte de los trabajos de investigación se han centrado en analizar de manera dimensional las variables de capital social y capacidad de absorción, dentro de las cuales se encuentran las variables en estudio como dimensiones. Como primera contribución del trabajo, se demostró empíricamente que existe una relación directa entre el capital relacional y el crecimiento empresarial; es decir, el capital relacional juega un rol importante en el crecimiento empresarial, sugiriendo que la confianza, amistad y respeto promueven mejores vínculos comerciales y de cooperación.

Segundo, se determinó que el rol de la capacidad de asimilación del conocimiento, además de influenciar directamente en el crecimiento empresarial, sirve como medio a través del cual el capital relacional incrementa su influencia en el crecimiento empresarial a partir del conocimiento adquirido externamente de las personas y organizaciones del microentorno competitivo, que al ser asimilado a través de innovaciones, asegura el crecimiento empresarial. En otras palabras, a mayor asimilación del conocimiento interno y externo se tendrá mejores resultados.

Además, como una implicancia teórica y práctica, se sugiere que estas relaciones deben darse de manera sincrónica para aprovechar la sinergia de estas variables. En concreto, los gerentes de las empresas del sector textil deben de fomentar relaciones con sus clientes, proveedores y stakeholders en un ambiente de confianza, amistad y respeto. Este entorno de relaciones virtuosas ayudará a las organizaciones a asimilar el conocimiento existente, interno y externo, al promover su análisis, procesamiento, comprensión. Además, este conocimiento se verá reflejado en el desarrollo de nuevos procesos y productos con los cuales se sustenta el crecimiento empresarial.

A pesar de las previsiones tomadas en el desarrollo de la investigación, no está libre de limitaciones; y, si bien es cierto que se han realizado pruebas para validar las escalas utilizadas, es posible que no se hayan eliminado posibles sesgos. Otra limitación es el espacio del estudio: las micro y pequeñas empresas de la región Arequipa, en Perú, aspecto que podría limitar la generalización de los resultados. Sin embargo, cabe señalar que estos sí podrían validarse en otros países en desarrollo.

Se recomienda que a futuro se realicen estudios más detallados de las diferentes dimensiones del capital social y la capacidad de absorción, con el objeto de que actúen de manera indirecta y favorezcan el desarrollo empresarial. 


\section{Apéndice}

Tabla 8. Escala de medición del capital relacional

\begin{tabular}{|c|c|c|c|c|c|c|c|}
\hline Ítems & & & duI & nte & a & & \\
\hline $\begin{array}{l}\text { Tenemos relaciones estrechas y personales con nuestros contac- } \\
\text { tos }\end{array}$ & 1 & 2 & 3 & 4 & 5 & 6 & 7 \\
\hline $\begin{array}{l}\text { Las relaciones con nuestros contactos se caracterizan por un } \\
\text { respeto mutuo }\end{array}$ & 1 & 2 & 3 & 4 & 5 & 6 & 7 \\
\hline $\begin{array}{l}\text { Las relaciones con nuestros contactos se caracterizan por una } \\
\text { confianza mutua }\end{array}$ & 1 & 2 & 3 & 4 & 5 & 6 & 7 \\
\hline $\begin{array}{l}\text { Las relaciones con nuestros contactos se caracterizan por una alta } \\
\text { reciprocidad }\end{array}$ & 1 & 2 & 3 & 4 & 5 & 6 & 7 \\
\hline
\end{tabular}

Fuente: adaptado de García-Villaverde et al. (201) y Rodrigo-Alarcón et al. (2018).

Tabla 9. Escala de medición de la capacidad de absorción

\begin{tabular}{l|l|l|l|l|l|l|c}
$\begin{array}{l}\text { Por favor, indique su grado de acuerdo o desacuerdo con las } \\
\text { siguientes afirmaciones referidas a las capacidades de su } \\
\text { empresa }\end{array}$ & \multicolumn{5}{|c}{1 = totalmente en desacuerdo } \\
\hline $\begin{array}{l}\text { En nuestra empresa las ideas y conceptos son comunicados entre } \\
\text { los departamentos }\end{array}$ & 1 & 2 & 3 & 4 & 5 & 6 & 7 \\
\hline $\begin{array}{l}\text { Nuestros directivos hacen hincapié en el apoyo interdepartamental } \\
\text { para resolver los problemas }\end{array}$ & 1 & 2 & 3 & 4 & 5 & 6 & 7 \\
\hline $\begin{array}{l}\text { En nuestra empresa hay un flujo rápido de información; por } \\
\text { ejemplo, si un departamento obtiene información importante, la } \\
\text { comunica inmediatamente a todos los demás }\end{array}$ & 1 & 2 & 3 & 4 & 5 & 6 & 7 \\
\hline $\begin{array}{l}\text { Nuestros directivos piden reuniones interdepartamentales periódi- } \\
\text { cas para intercambiar nuevos desarrollos, problemas y logros }\end{array}$ & 1 & 2 & 3 & 4 & 5 & 6 & 7
\end{tabular}

Fuente: adaptado de García-Villaverde et al. (2018) y Parra-Requena et al. (2015).

Tabla 10. Escala de medición del resultado empresarial

\begin{tabular}{l|c|c|c|c|c|c|c|c|c|c|c|c|c|c}
\multicolumn{7}{|c|}{} & \multicolumn{7}{|c}{ Importancia } & \multicolumn{7}{c}{ Satisfacción } \\
\hline (RE3) Cuota de mercado & 1 & 2 & 3 & 4 & 5 & 6 & 7 & 1 & 2 & 3 & 4 & 5 & 6 & 7 \\
\hline (RE4) Crecimiento de ventas & 1 & 2 & 3 & 4 & 5 & 6 & 7 & 1 & 2 & 3 & 4 & 5 & 6 & 7
\end{tabular}

Fuente: adaptado de García-Villaverde et al. (2014). 


\section{bibliografía}

Andrade, Y., Cardenas, L.,

Viacava, G., Raymundo, C., \&

Dominguez, $F$.

2019 Lean manufacturing model for the

reduction of production times and

reduction of the returns of defective

items in textile industry. En Advances

in Design for Inclusion. Proceedings of

the AHFE 2019 International Conference

on Design for Inclusion and the AHFE

2019 International Conference on Human

Factors for Apparel and Textile Engineering

(pp. 387-398). Springer. https://doi.

org/10.1007/978-3-030-20444-0_39

Arshad, M. Z., \& Arshad, D.

2019 Internal capabilities and SMEs

performance: A case of textile industry in

Pakistan. Management Science Letters,

9(4), 621-628. https://doi.org/10.5267/j.

msl.2019.1.001

\section{Avkiran, N. K.}

2018 An in-depth discussion and illustration of partial least squares structural equation modeling in health care. Health Care Management Science, 21(3), 401-408. https://doi.org/10.1007/s10729-017-9393-7

\section{Bunclark, L., \& Barcellos-Paula, L.}

Sustainability reporting for sustainable

supply chain management in Peru.

Sustainable Production and Consumption,

27, 1458-1472. https://doi.org/10.1016/j.

spc. 2021.03 .013

\section{Carmines, E. G., \& Zeller, R. A.}

2010

$$
\text { Camisón, C., \& Forés, B. }
$$

Knowledge absorptive capacity: New insights for its conceptualization and measurement. Journal of Business Research, 63(7), 707-715. https://doi. org/10.1016/j.jbusres.2009.04.022

\section{Cepeda, G., Nitzl, C., \& Roldán, J.}

2017

Mediation Analyses in Partial Least

Squares Structural Equation Modeling:

Guidelines and Empirical Examples. Partial

Least Squares Path Modeling: Basic

Concepts, Methodological Issues and

Applications (pp. 173-195). https://doi.

org/10.1007/978-3-319-64069-3

\section{Chin, W. W.}

1998

The Partial Least Squares Approach to Structural Equation Modeling. En G. A. Marcoulides (ed.), Modern methodsfor business research (pp. 295-358). California: Lawrence Erlbaum.

Cohen, W. M., \& Levinthal, D. A.

Absorptive Capacity: A New Perspective on Learning and Innovation. Administrative Science Quarterly, 35(1), 128-152. https:// doi.org/10.2307/2393553

Cortez, C., Di Laura, N., Viacava, G., Raymundo, C., \&

\section{Dominguez, $\mathrm{F}$.}

\section{Lean Manufacturing Model Based on}

Knowledge Management to Increase

Compliance in the Production Process

in Peruvian SMEs in the Textile Garment

Sector. En Advances in Human Factors,

Business Management and Leadership

Proceedings of the AHFE 2019

International Conference on Human

California: Sage. https://dx.doi.

org/10.4135/9781412985642 


\section{bibliografía}

Factors, Business Management and

Society, and the AHFE International

Conference on Human Factors in

Management and Leadership (pp. 103-111).

Springer. https://doi.org/10.1007/978-3-030-

20154-8_10

Debrulle, J., Maes, J., \& Sels, L.

2013

Start-up absorptive capacity: Does

the owner's human and social capital

matter? International Small Business

Journal: Researching Entrepreneurship,

32(7), 777-801. https://doi.

org/10.1177/0266242612475103

Dobbs, M., \& Hamilton, R. T.

2007

Small business growth: recent evidence

and new directions. International Journal

of Entrepreneurial Behaviour and

Research, 13(5), 296-322. https://doi.

org/10.1108/13552550710780885

\section{Expósito-Langa, M., Molina-}

Morales, F. X., \& Tomás-Miquel,

J. V.

2015

How shared vision moderates the

effects of absorptive capacity and

networking on clustered firms' innovation.

Scandinavian Journal of Management,

31(3), 293-302. https://doi.org/10.1016/j.

scaman.2015.06.001

Flatten, T. C., Engelen, A., Zahra,

\section{S. A., \& Brettel, M.}

2011 A measure of absorptive capacity: Scale

development and validation. European

Management Journal, 29(2), 98-116.

https://doi.org/10.1016/j.emj.2010.11.002

\section{Fornell, C., \& Larcker, D. F.}

2010

(a)

El papel mediador de las capacidades tecnológicas y de marketing. Cuadernos de Economía y Dirección de la Empresa, 13(45), 9-42. https://doi.org/10.1016/s11385758(10)70022-0

\section{García-Villaverde, P. M., Ruiz- Ortega, M. J., Parra-Requena, G., \& Rodrigo-Alarcón, J.}

Orientación emprendedora y resultado empresarial: implicaciones de los factores relacionales. Cuadernos Aragoneses de Economía, 24(1-2), 75-96.

García-Villaverde, P. M., RodrigoAlarcón, J., Ruiz-Ortega, M. J., \& Parra-Requena, G.

The role of knowledge absorptive capacity on the relationship between cognitive social capital and entrepreneurial orientation. Journal of Knowledge Management, 22(5), 1015-1036. https://doi. org/10.1108/JKM-07-2017-0304

\section{Gielnik, M. M., Zacher, H., \&} Schmitt, A.

How Small Business Managers' Age and Focus on Opportunities Affect Business Growth: A Mediated Moderation Growth Model. Journal of Small Business Management, 55(3), 460-483. https://doi. org/10.1111/jsbm. 12253 


\section{bibliografía}

Hair, J. F., Hult, G. T. M., Ringle, C. M., Sarstedt, M., \& Thiele, K. O.

2017 Mirror, mirror on the wall: a comparative evaluation of composite-based structural equation modeling methods. Journal of the Academy of Marketing Science, 45(5), 616-632. https://doi.org/10.1007/s11747017-0517-x

\section{Hair, J. F., Risher, J. J., Sarstedt,} M., \& Ringle, C. M.

2019 When to use and how to report the results of PLS-SEM. European Business Review, 31(1), 2-24. https://doi.org/10.1108/EBR-112018-0203

\section{Henseler, J., Ringle, C. M., \& Sarstedt, M.}

2015 A new criterion for assessing discriminant validity in variance-based structural equation modeling. Journal of the Academy of Marketing Science, 43(1), 115-135. https://doi.org/10.1007/s11747014-0403-8

\section{Hjalager, A. M.}

2010 A review of innovation research in tourism. Tourism Management, 31(1), 1-12. https:// doi.org/10.1016/j.tourman.2009.08.012

Hughes, M., Morgan, R., Ireland, D., \& Hughes, P.

Social Capital and Learning Advantages: A Problem of Absorptive Capacity. Strategic Entrepreneurship Journal, 8(3), 214-233. https://doi.org/10.1002/sej.1162

\section{Israel, A., \& Hitzeroth, M.}

How do micro- and small-scale enterprises respond to global competition? An example of the textile survival cluster Gamarra in Lima. International Development Planning
Review, 40(2), 203-222. https://doi. org/10.3828/idpr.2018.9

Jeong, S. W., Chung, J. E., \& Roh, J. S.

2019

Impact of External Knowledge Inflow on Product and Process Innovation of Korean SMEs: Absorptive Capacity as a Mediator. Clothing and Textiles Research Journal, 37(4), 219-234. https://doi. org/10.1177/0887302X19860913

Kohtamäki, M., Heimonen, J., \& Parida, V.

2019 The nonlinear relationship between entrepreneurial orientation and sales growth: The moderating effects of slack resources and absorptive capacity. Journal of Business Research, 100, 100-110. https://doi.org/10.1016/j. jbusres.2019.03.018

\section{Kor, Y., \& Mahoney, J.}

2004 Edith Penrose's Contributions to the Resource-based View of Strategic Management Yasemin. Journal of Management Studies, 41(1), 183191. https://doi.org/10.1111/j.14676486.2004.00427.x

\section{Latan, H., \& Noonan, R.}

2017 Partial Least Squares Path Modeling. Basic Concepts, Methodological Issues and Applications. Springer. https://doi. org/10.1007/978-3-319-64069-3

Liu, X., Zhao, H., \& Zhao, X.

2018 Absorptive capacity and business performance: The mediating effects of innovation and mass customization. Industrial Management \& Data Systems, 118(9), 1787-1803. https://doi.org/10.1108/ IMDS-09-2017-0416 


\section{bibliografía}

Martins, J.T.

2016

Relational capabilities to leverage

new knowledge: Managing directors'

perceptions in UK and Portugal old

industrial regions. Learning Organization,

23(6), 398-414. https://doi.org/10.1108/TLO-

03-2016-0022

\section{Ministerio de producción} (Produce)

Estudio de investigación del sector textil y confecciones. Lima.

Miroshnychenko, I., De Massis, A., Miller, D., \& Barontini, R.

Family Business Growth Around the

World. Entrepreneurship: Theory and

Practice, 45(4), 682-708. https://doi.

org/10.1177/1042258720913028

Mubarik, M. S., Naghavi, N., \&

Mubarak, M. F.

2019

Impact of Supplier Relational Capital on

Supply Chain Performance in Pakistani

Textile Industry. Asian Economic and

Financial Review, 9(3), 318-328. https://doi.

org/10.18488/journal.aefr.2019.93.318.328

\section{Murale, V., Sisodia, G. S., \&}

Preetha, R.

2018

Business relational capital and firm

performance: an insight from Indian textile industry. International Journal of Learning and Intellectual Capital, 15(4), 341-362.

https://doi.org/10.1504/IJLIC.2018.095899

\section{Nahapiet, J., \& Ghoshal, S.}

Nitzl, C., Roldan, J. L., \&

Cepeda-Carrión, $\mathbf{G}$.

2016

Mediation analysis in partial least squares

path modeling: Helping researchers

discuss more sophisticated models.

Industrial Management \& Data Systems,

116(9), 1849-1864. https://doi.org/10.1108/

IMDS-07-2015-0302

\section{Ødegaard, C. V.}

Made in China'. Contraband, Labour and

the Gendered Effects of 'Free-Trade',

China-Peru. Journal of Development

Studies, 53(3), 346-360. https://doi.org/10.1

080/00220388.2016.1184249

Parra-Requena, G., Ruiz-Ortega,

M. J., García-Villaverde, P. M., \& Rodrigo-Alarcón, J.

2015

The Mediating Role of Knowledge Acquisition

on the Relationship between External

Social Capital and Innovativeness. European

Management Review, 12(3), 149-169. https://

doi.org/10.1111/emre.12049

Partanen, J., Möller, K.,

Westerlund, M., Rajala, R., \&

Rajala, A.

2008

Social capital in the growth of scienceand-technology-based SMEs. Industrial

Marketing Management, 37(5),

513-522. https://doi.org/10.1016/j.

indmarman.2007.09.012

\section{Penrose, E.}

The Theory of the Firm. Nueva York: John Wiley \& Sons.

Prajogo, D., Mena, C., \& Chowdhury, $\mathrm{M}$. 


\section{bibliografía}

product performance - a moderated-

mediated model. International Journal of

Operations \& Production Management,

41(3), 206-226. https://doi.org/10.1108/

IJOPM-05-2020-0256

\section{Rigdon, E. E.}

Rethinking Partial Least Squares Path

Modeling: In Praise of Simple Methods.

Long Range Planning, 45(5-6), 341-358.

https://doi.org/10.1016/j.Irp.2012.09.010

Roberts, N., \& Thatcher, J. B.

2009

Conceptualizing and Testing Formative

Constructs: Tutorial and Annotated

Example. Data Base for Advances in

Information Systems, 40(3), 9-39. https://

doi.org/10.1145/1592401.1592405

\section{Rodrigo-Alarcón, J.}

2013 Social capital and entrepreneurial orientation in the agri-food industry: The mediating role of dynamic capabilities [tesis doctoral, Universidad de Castilla La Mancha]. https://www.educacion. gob.es/teseo/imprimirFicheroTesis. do?idFichero=gM1RkV8KSp4\%3D

\section{Rodrigo-Alarcón, J., García-} Villaverde, P. M., Ruiz-Ortega, M. J., \& Parra-Requena, G.

2018 From social capital to entrepreneurial orientation: The mediating role of dynamic capabilities. European Management Journal, 36(2), 195-209. https://doi. org/10.1016/j.emj.2017.02.006

Rodrigo-Alarcón, J., ParraRequena, G., \& GarcíaVillaverde, P. M.

Efectividad de la orientación emprendedora: el papel del capital social y las capacidades. Investigaciones Europeas de Dirección y Economía de la Empresa, 20(3), 131-139. https://doi.org/10.1016/j. iedee.2013.09.002

\section{Rodrigo-Alarcón, J., Parra-}

\section{Requena, G., \& Ruiz-Ortega,}

M. J.

2020

Cognitive social capital and absorptive capacity as antecedents of entrepreneurial orientation: a configurational approach.

Eurasian Business Review, (10), 493-517.

https://doi.org/10.1007/s40821-020-00169-3

\section{Roldan, J. L., \& Sanchez- \\ Franco, M. J.}

2012 Chapter 10 Variance - Based Structural Equation Modeling: Guidelines for Using Partial Least Squares in Information Systems Research. En Research Methodologies, Innovations and Philosophies in Software Systems Engineering and Information Systems (pp. 193-221). https://doi. org/10.4018/978-1-4666-0179-6

\section{Safrida, S., Suprayitno, E.,} Tarigan, N. M. R., \& Wardhani, I. I.

Absorptive capacity: As a mediator between social capital and supply chain resilience in the textile industry of Thailand International Journal of Supply Chain Management, 9(4), 310-316.

\section{Superintendencia Nacional de} Aduanas y de Administración Tributaria (Sunat)

Solicitud de Acceso a la Información
Pública - Ley No 27806. https://
www.sunat.gob.pe/cuentassunat/
informacionContribuyente/index.html




\section{bibliografía}

Yoo, S. J., Sawyerr, O., \& Tan,

W. L.

2016 The Mediating Effect of Absorptive

Capacity and Relational Capital in Alliance

Learning of SMEs. Journal of Small

Business Management, 54(51), 234-255.

https://doi.org/10.1111/jsbm.12299

Yu, Y., \& Huo, B.

2019 The impact of relational capital on supplier quality integration and operational performance. Total Quality Management and Business Excellence, 30(11-12), 12821301. https://doi.org/10.1080/14783363.20 17.1366265

Yu, Y., Zhang, M., \& Huo, B.

2021 The impact of relational capital on green supply chain management and financial performance. Production Planning and Control, 32(10), 861-874. https://doi.org/10.1 080/09537287.2020.1774675

Fecha de recepción: 14 de diciembre de 2020 Fecha de aprobación: 2 de agosto de 2021 Fecha de publicación: 20 de setiembre de 2021 